Revista Internacional de Ciencias Podológicas

ISSN: 1989-5151

http://dx.doi.org/10.5209/RICP.57989

\title{
Tratamiento quirúrgico frente al conservador para la ruptura aguda del tendón de Aquiles
}

Patricia López Olivera ${ }^{1}$.

Fechas: Fecha de recepción: 30 de marzo de 2016 / Fecha de aceptación: 2 de junio de 2016

\section{Resumen.}

Introducción: La reparación quirúrgica es un método común para el tratamiento de la ruptura aguda del tendón de Aquiles, ya que, a pesar de presentar un mayor riesgo de complicaciones, ofrece un menor riesgo de rotura recurrente. Sin embargo, análisis recientes basados en el empleo de rehabilitación funcional temprana, han cuestionado esta creencia. El objetivo de este estudio consiste en evaluar la efectividad clínica de ambos tratamientos.

Metodología: Se han empleado cuatro bases de datos (Medline, CINAHL, PubMed y Cochrane Library) para la búsqueda de estudios que comparen el tratamiento quirúrgico y el conservador para la ruptura aguda del tendón de Aquiles. La selección de los artículos se realizó en función de los criterios de inclusión y exclusión.

Resultados: Los estudios mostraron que el tratamiento quirúrgico presenta una tasa inferior de rotura recurrente $(\mathrm{P}=0.002)$ en comparación con el conservador, pero aumenta el riesgo de otras complicaciones $(\mathrm{P}=0.004)$. Un análisis de subgrupos reveló una tasa significativamente más alta en relación con la adherencia cicatricial $(\mathrm{P}<0.00001)$, la infección superficial $(\mathrm{P}=0.05)$ y la perturbación de la sensibilidad $(\mathrm{P}=0.0003)$ en el grupo sometido a cirugía.

Conclusiones: El tratamiento quirúrgico, en comparación con el conservador, puede reducir eficazmente el riesgo de rotura recurrente, pero incrementa la probabilidad de complicaciones, lo cual suele estar asociado a la cirugía de reparación abierta. No obstante, no hay pruebas suficientes de estudios actuales que apoyen la creencia de que la cirugía puede conducir a una mejor recuperación funcional.

Palabras clave: ruptura aguda tendón Aquiles; tratamiento conservador; tratamiento quirúrgico.

\section{[en] Surgical versus Conservative Treatment for Acute Achilles Tendon Rupture}

\begin{abstract}
.
Background: Surgical repair is a common method of treatment of acute Achilles tendon rupture because, despite a higher risk of overall complications, it has been believed to offer a reduced risk of rerupture. However, recent analysis based on using early functional rehabilitation, have challenged this belief. The aim of this study is to evaluate the clinical effectiveness of both treatments.

Methods: The search was performed using the following electronic bibliography databases (Medline, CINAHL, PubMed and Cochrane Library) to find studies comparing surgical and conservative treatment for acute Achilles tendon rupture. The selection of items was based on inclusion and exclusion criteria.

Results: The studies showed that surgical treatment has a lower rate of rerupture $(P=0.002)$ compared with nonsurgical treatment, but increases the risk of other complications $(P=0.004)$. Subgroup analyses revealed significant differences in relation to scar adhesion $(P<0.00001)$, superficial infection $(P=0.05)$ and sensibility disturbance $(P=0.0003)$ in the group undergoing surgery.

Conclusions: Compared with conservative treatment, operative treatment can effectively reduce the risk of rerupture but increase the probability of complications, which is usually associated with open repair surgery. However, no sufficient evidence is available from current studies to support the belief that operation may lead to better functional recovery.

Key words: acute Achilles tendon rupture; conservative treatment; operative treatment.
\end{abstract}

La autora declara no tener ningún tipo de interés económico o comercial.

Colaboradora. Podología General.Facultad de Enfermería, Fisioterapia y Podología. Universidad Complutense de Madrid. patricialopezolivera@hotmail.com 
Sumario: 1. Introducción. 2. Desarrollo. 2.1. Material y métodos. 2.1.1. Desarrollo de la pregunta clínica. 2.2.2. Resultados de la estrategia de búsqued. 3. Resultados. 3.1. Descripción de los estudios. 4. Discusión. 5. Conclusiones. 6. Bibliografía. 7. Anexos.

Cómo citar: López Olivera, P. (2018) Tratamiento quirúrgico frente al conservador para la ruptura aguda del tendón de Aquiles, en Revista internacional de ciencias podológicas 12(1), 45-54.

\section{Introducción}

El tendón de Aquiles es el tendón más largo $\mathrm{y}$ fuerte del cuerpo humano, pero también es el que se rompe con mayor frecuencia ${ }^{1,2}$. De acuerdo con un informe reciente en Finlandia $^{3}$, la incidencia de roturas agudas del tendón de Aquiles aumentó de 8.3/105 en 1987 a $14.8 / 105$ en 1999 y se cree que está en aumento; además se presentan con mayor frecuencia en grupos de hombres y mujeres de 30-39 y 40-49 años de edad ${ }^{4}$, con predominio masculino ${ }^{5}$. De media, las mujeres con rotura del tendón tienden a ser de 2 a 3 años mayores que los hombres $(3,4)$.

Muchos estudios reportan que la ruptura aguda del tendón de Aquiles se produce principalmente durante las actividades deportivas $^{6-10}$, especialmente en juegos de raqueta, fútbol y balonmano, ${ }^{6,7,10,11}$. El mecanismo de lesión incluye un repentino empujón de la parte delantera del pie que soporta el peso con la rodilla en extensión, una dorsiflexión inesperada y una violenta dorsiflexión de un pie en flexión plantar ${ }^{12}$.

En cuanto a la etiología, han sido propuestos muchos factores posibles ${ }^{13}$, tales como una pobre vascularización del tendón y degeneración del mismo ${ }^{13,14}$, disfunción gastro-sólea $^{13}$, efectos adversos de algunos fármacos (por ejemplo corticoesteroides ${ }^{15,16} \mathrm{y}$ fluoroquinolonas ${ }^{17,18}$ ) y la hipertermia inducida por el ejercicio ${ }^{19,20}$.

El diagnóstico se realiza basado en la palpación de un hueco y en una prueba de Thompson positiva, aunque si el examen físico es equívoco, la ecografía o la resonancia magnética se pueden emplear para confirmarlo $3,21,22$.

Las opciones de tratamiento incluyen el manejo conservador y quirúrgico: si el médico que lo trata opta por el no quirúrgico, el paciente es tratado con un molde de yeso o con una férula con el pie situado en flexión plantar, con o sin fisioterapia temprana; mientras que las opciones quirúrgicas incluyen la reparación abierta, la técnica mínimamente invasiva y la reparación percutánea del tendón ${ }^{23}$.

En general, se acepta que la operación se debe realizar en atletas y pacientes jóvenes y en forma, y que el tratamiento conservador puede ser adecuado para las personas de edad ${ }^{24,25}$. Sin embargo, la controversia se mantiene con respecto al tratamiento óptimo para la ruptura aguda del tendón de Aquiles, ya que ambos presentan pros y contras ${ }^{6,7,26-29}$.

Los defensores del tratamiento no quirúrgico argumentan que mantener el pie en flexión plantar es suficiente para permitir la cicatrización adecuada del tendón, ya que en teoría, la curación se logra sin el alto riesgo de complicaciones asociado al tratamiento quirúrgico, las cuales ocurren hasta en el 10\% de los casos e incluyen infección profunda, necrosis de la piel, necrosis del tendón y un seno drenante. La prevalencia de complicaciones menores es también sustancial, con hasta un $15 \%$ de pacientes que desarrollan problemas de la piel $^{3,21-23,30}$.

La mayoría de cirujanos que optan por la reparación quirúrgica del tendón de Aquiles, lo hacen porque el tratamiento conservador está asociado a una tasa inaceptable de rotura recurrente; de hecho, según algunos estudios, dicha tasa va del $10 \%$ al $12 \%$, mientras que la cirugía disminuye la tasa por debajo del $3 \%$. Por tanto, el riesgo de complicaciones asociadas a la cirugía parece ser un compromiso aceptable para los pacientes y los cirujanos que persiguen este tratamiento ${ }^{3,21-23}$.

Tradicionalmente, el miembro afectado se ha inmovilizado por un mínimo de seis a ocho semanas antes de iniciar los ejercicios de rango de movimiento, cuyo objetivo es proteger la reparación quirúrgica (si se elige el tratamiento quirúrgico) o mantener la aposición del tendón (si se elige el tratamiento conservador); sin embargo, más recientemente, los refuerzos funcionales y los regímenes postoperatorios modificados permiten a los pacientes llevar a cabo diariamente ejercicios activos de flexión plantar diez días después de la lesión, lo cual 
está pensado para estimular la cicatrización del tendón y también puede disminuir la tasa de re-ruptura típicamente asociada al tratamiento conservador $^{31}$.

El objetivo de este estudio consiste en comprar el abordaje quirúrgico y el conservador con respecto a la tasa de rotura recurrente, la tasa global de otras complicaciones, el regreso al trabajo, la fuerza, el diámetro de la pantorrilla y los resultados funcionales, así como explorar los efectos de la rehabilitación funcional temprana.

Por este motivo, puede resultar de gran interés científico la elección del tema a la hora de optar por una u otra técnica, ya que la literatura basada en la evidencia actual determina las ventajas y desventajas de ambos tratamientos.

\section{Desarrollo}

\subsection{Material y métodos}

\subsubsection{Desarrollo de la pregunta clínica}

La pregunta clínica para esta revisión sistemática se generó utilizando el formato PICO, de la siguiente forma: "En pacientes con ruptura aguda del tendón de Aquiles, ¿qué tratamiento es más eficaz a la hora de disminuir el riesgo de rotura recurrente?".

P: paciente o patología: ruptura aguda del tendón de Aquiles.

I: intervención, es decir, tipo de tratamiento que se desea investigar: tratamiento conservador.

C: comparación, es decir, otros tipos de tratamientos que se desean comparar: tratamiento quirúrgico.

O: resultados, es decir, tipos de resultados que se han obtenido: el tratamiento conservador presenta una alta tasa de recurrencia frente al tratamiento quirúrgico.

Esta pregunta se realizó mediante diferentes términos de búsqueda para los cuales se emplearon cuatro bases de datos (Medline, CINAHL, Pubmed y Cochrane Library) (Tabla $1)$.

\subsubsection{Resultados de la estrategia de búsqueda}

Se revisaron de forma independiente todos los artículos encontrados en función de los criterios de elegibilidad (Tabla 2).

Solo fueron seleccionados aquellos artículos en inglés y a texto completo que comparasen el tratamiento quirúrgico con el conservador (Figura 1). Se excluyeron estudios retrospectivos, estudios de cohorte y estudios clínicos controlados. Los criterios de elegibilidad fueron: pacientes adultos con un claro diagnóstico de ruptura aguda del tendón de Aquiles (prueba de Thompson positiva, presencia de un hueco palpable, pérdida de fuerza de flexión plantar), tratamientos quirúrgicos basados en cirugía abierta o en técnicas mínimamente invasivas y tratamiento conservador a través de yesos o férulas.

Los criterios de exclusión fueron: pacientes tratados por roturas recurrentes, heridas abiertas y artículos repetitivos.

\section{Resultados}

\subsection{Descripción de los estudios}

Se realiza un resumen de los artículos que han sido empleados comprendido en la Tabla 3.

En el estudio de Jiang y col. ${ }^{32}$ fueron seleccionados un total de 894 pacientes con una edad media de 41 años, los cuales se dividieron en dos grupos: uno tratado mediante tratamiento quirúrgico y otro mediante tratamiento conservador, diferenciando entre hombres y mujeres y el miembro lesionado (lado derecho/lado izquierdo). El tiempo medio de seguimiento en un rango de 1-5 años fue de 2.5 , y en un rango de 0.5-1 años fue de 0.67 .

19 pacientes $(4,31 \%)$ de los 441 pertenecientes al grupo de tratamiento quirúrgico sufrieron roturas recurrentes en comparación con los 44 pacientes $(9,71 \%)$ de los 453 que pertenecían al grupo de tratamiento conservador, suponiendo una diferencia significativa entre ambos $(P=0.002)$.

En cambio, no existe una diferencia estadísticamente significativa entre los dos métodos en el número de pacientes que reanudaron el deporte tras la lesión $(P=0.30)$.

Sin embargo, los pacientes manipulados quirúrgicamente muestran una mayor tasa de 
complicaciones que los tratados de manera conservadora $[26.60 \%(112 / 421)$ vs. $7.19 \%$ (31/431)] $(P=0.004)$, y el tiempo medio de recuperación tras el tratamiento quirúrgico es menor que tras el tratamiento conservador $(P=0.009)$.

Se creó un subgrupo de meta-análisis para realizar comparaciones entre las complicaciones obtenidas: el grupo sometido a cirugía muestra una tasa significativamente más alta en la adherencia cicatricial $(P<0.00001)$, en la infección superficial $(P=0.05)$ y en la perturbación de la sensibilidad $(P=0.0003)$ que el grupo tratado de forma conservadora. Por el contrario, se han observado pocas diferencias significativas con respecto a la infección profunda $(P=0.22)$, la trombosis venosa profunda $(P=0.14)$ o el excesivo alargamiento del tendón de Aquiles $(P=0.31)$ en ambos tratamientos.

En el estudio de Soroceanu y col. ${ }^{33}$ se incluyeron un total de 826 pacientes, de los cuales 418 fueron intervenidos quirúrgicamente y 408 conservadoramente, con predominio del sexo masculino y una media de edad de cada grupo de 39,8 años.

\section{- Tasa de rotura recurrente:}

Los resultados en cuanto a la tasa de reruptura indican que la diferencia de riesgo entre ambos grupos es de un 5.5\% a favor de la cirugía $(P=0.002)$. Cabe destacar que hubo una significativa heterogeneidad entre los estudios, por lo que los factores que eran potencialmente responsables de ello fueron investigados.

El uso de rehabilitación funcional era una causa significativa de heterogeneidad $(P<0.05)$. El resto de factores que se consideraron (el tamaño del estudio, la duración del seguimiento y el año de publicación) no fueron significativos.

Por tanto, se realizó un análisis estratificado de las tasas de rotura recurrente según la rehabilitación funcional. Los resultados mostraron que siguiendo un protocolo mediante movilización temprana, la tasa entre ambos tratamientos era equivalente $(P=0.45)$, lo que sugiere que no se consiguió reducir el riesgo absoluto mediante la cirugía. Sin embargo, si el protocolo de tratamiento empleado después de la ruptura incluye inmovilización prolongada, la reducción absoluta del riesgo de rotura recurrente obtenido tras la intervención quirúrgica fue de un $8.8 \%$ $(P=0.001)$.

\section{- Otras complicaciones:}

En cuanto a otro tipo de complicaciones, tales como infecciones superficiales y profundas de la herida, necrosis de la piel y del tendón, fístulas, la adherencia cicatricial, daño del nervio sural, la disminución de la movilidad del tobillo, el excesivo alargamiento del tendón o la trombosis venosa profunda, los resultados indicaron que la diferencia de riesgo era de un $15.8 \%$ a favor del tratamiento conservador $(P=0.016)$.

\section{- Tiempo de retorno al trabajo:}

Los datos muestran que aquellos pacientes cuya ruptura del tendón fue reparada quirúrgicamente volvieron al trabajo 19.16 días antes que los que se sometieron al tratamiento conservador $(P=0.0014)$.

\section{- Amplitud de movimiento:}

Los estudios revelaron que la diferencia de grados de flexión plantar activa de tobillo en comparación con el lado contralateral era de $1.07^{\circ}$ menos en pacientes sometidos a tratamiento conservador $(P=0.019)$. Aunque estos resultados son estadísticamente significativos, no representan una diferencia clínicamente importante.

\section{- Diámetro de la pantorrilla:}

Los análisis no mostraron una diferencia significativa en cuanto al diámetro de la pantorrilla (medido en centímetros) en comparación con el miembro contralateral entre los dos grupos $(P=0.357)$.

\section{- Fuerza:}

Los resultados de los análisis mostraron que no hubo una diferencia significativa entre ambos grupos en relación con la media estandarizada $(P=0.806)$.

\section{- Resultados funcionales:}

Los datos obtenidos sobre los resultados funcionales, expresados mediante diferentes escalas (Instrumento de Evaluación Funcional Músculo-esquelética [MFAI], Índice Funcional de la Pierna y el Tobillo [FIL], el marcador Leppilahti y el marcador Leppilahti modificado) mostraron que la media estandarizada no difería significativamente entre los dos grupos $(P=0.226)$. 


\section{Discusión}

Los resultados de los estudios ${ }^{32}$ confirman que en el tratamiento de la ruptura aguda del tendón de Aquiles, la cirugía puede reducir eficazmente el riesgo de rotura recurrente, pero es difícil evitar una alta tasa de complicaciones.

En primer lugar, llama la atención que los hombres representan el 81,52\% de todos los pacientes. Este predominio masculino $(4,41: 1)$ puede indicar que los que participan en actividades de mayor violencia son, en su mayoría, hombres.

En segundo lugar, la mayor parte de ellos estaban entre los treinta y los cuarenta años, lo cual sugiere que la población de mediana edad sufre una pérdida de resistencia progresiva del tendón.

La literatura actual indica que los pacientes tratados mediante tratamiento conservador tienden a presentar una tasa más alta de reruptura. Esta diferencia estadísticamente significativa puede explicarse por dos factores: después de la curación sin sutura quirúrgica, los tendones una vez rotos no soportan bien la intensidad de las actividades como antes de la lesión, y el periodo habitual de inmovilización de 6-8 semanas del tratamiento conservador parece insuficiente para permitir la recuperación del tendón.

Otro estudio ${ }^{33}$ sugiere que los resultados entre ambos tratamientos en cuanto a la tasa de rotura recurrente eran equivalentes cuando se incluye movilización temprana en el protocolo de tratamiento conservador. Sin embargo, si no se emplea rehabilitación funcional, la reparación quirúrgica reduce el riesgo de reruptura un $8,8 \%$.

Por tanto, el tratamiento conservador representa una opción razonable en los centros que utilizan rehabilitación funcional mediante movilización temprana, mientras que la reparación quirúrgica puede estar indicada en los centros que no emplean movilización temprana o en aquellos pacientes que no son buenos candidatos para este protocolo, ya que, aunque está asociada a una mayor tasa global de otras complicaciones, redujo la tasa de rotura recurrente.

Un análisis de subgrupos ${ }^{27,28}$ muestra que la adherencia cicatricial, la infección superficial y la perturbación de la sensibilidad se asocian potencialmente con la cirugía de reparación abierta, por lo que los resultados revelaron diferencias significativas entre los dos tipos de tratamientos, pero no en cuanto a la infección profunda, la trombosis venosa profunda o el excesivo alargamiento del tendón. Por otro lado, los pacientes tratados quirúrgicamente pueden tener un tiempo medio de baja por enfermedad más corto que aquellos tratados de forma conservadora, es decir, la cirugía puede dar lugar a una recuperación más rápida, aunque existen discrepancias en cuanto a los resultados funcionales específicos. De este modo, el riesgo de otras complicaciones en los pacientes tratados quirúrgicamente era 3.9 veces mayor que los tratados conservadoramente.

Los pacientes sometidos a la cirugía volvieron al trabajo diecinueve días antes, de media, que los que fueron tratados de forma conservadora. Sin embargo, estos resultados están basados en un número pequeño de estudios, los cuales no informan sobre qué criterios se utilizaron para determinar cuándo un paciente debe reanudar las actividades laborales.

La diferencia en la flexión plantar activa entre ambos grupos no era clínicamente importante, y no hubo una diferencia significativa entre los dos tratamientos en cuanto al diámetro de la pantorrilla, la fuerza o los resultados funcionales.

El aumento del riesgo de tener una complicación que no sea una rotura recurrente en el grupo quirúrgico parece ser más importante que el aumento de riesgo de tener una rotura recurrente en el grupo conservador. Sin embargo, teniendo en cuenta que no todas las complicaciones son mayores, algunos pacientes y cirujanos consideran que este aumento después del tratamiento quirúrgico puede ser un compromiso aceptable para la tasa de rotura recurrente.

En cambio, los resultados obtenidos comparando ambos tratamientos podrían cambiar si se mejoran las terapias convencionales. Por ejemplo, se ha observado una asociación entre la cirugía de reparación abierta y los problemas en la herida.

Recientemente, las técnicas de reparación percutánea han sido adoptadas como nuevos procedimientos quirúrgicos que permiten reducir las complicaciones causadas por la cirugía abierta y han mostrado tener buena eficacia ${ }^{27}$.

Además, otros estudios ${ }^{34,35}$ probaron que la reparación percutánea del tendón de Aquiles no solo está indicada en pacientes diabéticos, 
sino que también es adecuada para aquellos mayores de 65 años.

En un meta-análisis reciente ${ }^{36}$ se concluyó que la cirugía percutánea mínimamente invasiva supone un riesgo significativamente menor de sufrir una infección superficial de la herida y un número tres veces mayor de pacientes obtuvieron resultados excelentes en comparación con la cirugía abierta convencional.

A su vez, otro estudio mostró que la reparación percutánea supone un tiempo de operación significativamente más corto que la cirugía abierta $(P<0.01)(37)$.

Del mismo modo, otros autores hicieron hincapié en las ventajas decisivas y la mayor eficacia del tratamiento conservador funcional (38).

\section{Conclusiones}

El tratamiento quirúrgico de la rotura aguda del tendón de Aquiles puede reducir eficazmente el riesgo de rotura recurrente, pero también puede dar lugar a un mayor número de complicaciones relacionadas con la cirugía abierta. Por otro lado, el tratamiento conservador puede presentar una tasa menor de complicaciones pero un alto riesgo de reruptura.

Basándonos en la evidencia actual, es difícil determinar cuál de los dos métodos es superior al otro, ya que se piensa que las mejoras de las técnicas en cualquiera de los dos tratamientos podrían cambiar sus ventajas y desventajas.

Por tanto, debe ampliarse la investigación y es necesario realizar más estudios que comparen la reparación percutánea con el tratamiento conservador para determinar las diferentes complicaciones entre las dos modalidades.

\section{Bibliografía}

1. Ames PRJ, Longo UG, Denaro V, Maffulli N. Achilles tendon problems: not just an orthopaedic issue. Disabil Rehabil [Internet]. 2008 Jan [cited 2015 Nov 19];30(20-22):1646-50. Available from: http:// www.ncbi.nlm.nih.gov/pubmed/18608383

2. Maffulli N, Waterston SW, Squair J, Reaper J, Douglas AS. Changing incidence of Achilles tendon rupture in Scotland: a 15-year study. Clin J Sport Med [Internet]. 1999 Jul [cited 2015 Nov 19];9(3):15760. Available from: http://www.ncbi.nlm.nih.gov/pubmed/10512344

3. Nyyssönen T, Lüthje P, Kröger H. The increasing incidence and difference in sex distribution of Achilles tendon rupture in Finland in 1987-1999. Scand J Surg [Internet]. 2008 Jan [cited 2015 Nov 19];97(3):272-5. Available from: http://www.ncbi.nlm.nih.gov/pubmed/18812279

4. Suchak AA, Bostick G, Reid D, Blitz S, Jomha N. The incidence of Achilles tendon ruptures in Edmonton, Canada. Foot ankle Int [Internet]. 2005 Nov [cited 2015 Sep 27];26(11):932-6. Available from: http://www.ncbi.nlm.nih.gov/pubmed/16309606

5. Hattrup SJ, Johnson KA. A review of ruptures of the Achilles tendon. Foot Ankle [Internet]. 1985 Aug [cited 2016 Feb 29];6(1):34-8. Available from: http://www.ncbi.nlm.nih.gov/pubmed/3899877

6. Nistor L. Surgical and non-surgical treatment of Achilles Tendon rupture. A prospective randomized study. J Bone Joint Surg Am [Internet]. 1981 Mar [cited 2015 Oct 7];63(3):394-9. Available from: http://www.ncbi.nlm.nih.gov/pubmed/7204438

7. Cetti R, Christensen SE, Ejsted R, Jensen NM, Jorgensen U. Operative versus nonoperative treatment of Achilles tendon rupture. A prospective randomized study and review of the literature. Am J Sports Med [Internet]. Jan [cited 2015 Nov 19];21(6):791-9. Available from: http://www.ncbi.nlm.nih.gov/ pubmed/8291628

8. Metz R, Verleisdonk E-JMM, van der Heijden GJ-M-G, Clevers G-J, Hammacher ER, Verhofstad MHJ, et al. Acute Achilles tendon rupture: minimally invasive surgery versus nonoperative treatment with immediate full weightbearing--a randomized controlled trial. Am J Sports Med [Internet]. 2008 Sep [cited 2016 Feb 29];36(9):1688-94. Available from: http://www.ncbi.nlm.nih.gov/pubmed/18645042

9. Thompson J, Baravarian B. Acute and chronic Achilles tendon ruptures in athletes. Clin Podiatr Med Surg [Internet]. 2011 Jan [cited 2016 Feb 29];28(1):117-35. Available from: http://www.ncbi.nlm.nih. gov/pubmed/21276522

10. Keating JF, Will EM. Operative versus non-operative treatment of acute rupture of tendo Achillis: a prospective randomised evaluation of functional outcome. J Bone Joint Surg Br [Internet]. 2011 Aug [cited 2015 Nov 19];93(8):1071-8. Available from: http://www.ncbi.nlm.nih.gov/pubmed/21768631 
11. Gwynne-Jones DP, Sims M, Handcock D. Epidemiology and outcomes of acute Achilles tendon rupture with operative or nonoperative treatment using an identical functional bracing protocol. Foot ankle Int [Internet]. 2011 Apr [cited 2015 Nov 19];32(4):337-43. Available from: http://www.ncbi.nlm.nih.gov/ pubmed/21733434

12. Arner O, Lindholm A. Subcutaneous rupture of the Achilles tendon; a study of 92 cases. Acta Chir Scand Suppl [Internet]. 1959 Jan [cited 2015 Nov 19];116(Supp 239):1-51. Available from: http://www. ncbi.nlm.nih.gov/pubmed/13660721

13. Maffulli N. Achilles tendon rupture. Br J Sports Med [Internet]. 1995 Dec [cited 2015 Nov 19];29(4):27980. Available from: http://www.pubmedcentral.nih.gov/articlerender.cgi?artid=1332246\&tool=pmcentrez\&rendertype $=$ abstract

14. Williams JG. Achilles tendon lesions in sport. Sports Med [Internet]. 1993 Sep [cited 2015 Nov 19];16(3):216-20. Available from: http://www.ncbi.nlm.nih.gov/pubmed/8235194

15. McQuillan R, Gregan P. Tendon rupture as a complication of corticosteroid therapy. Palliat Med [Internet]. 2005 Jun [cited 2015 Nov 19];19(4):352-3. Available from: http://www.ncbi.nlm.nih.gov/ pubmed/15984509

16. Kao NL, Moy JN, Richmond GW. Achilles tendon rupture: an underrated complication of corticosteroid treatment. Thorax [Internet]. 1992 Jun [cited 2015 Nov 19];47(6):484. Available from: http://www.pubmedcentral.nih.gov/articlerender.fcgi?artid $=463830 \&$ tool $=$ pmcentrez\&rendertype $=$ abstract

17. Seeger JD, West WA, Fife D, Noel GJ, Johnson LN, Walker AM. Achilles tendon rupture and its association with fluoroquinolone antibiotics and other potential risk factors in a managed care population. Pharmacoepidemiol Drug Saf [Internet]. 2006 Nov [cited 2015 Sep 27];15(11):784-92. Available from: http://www.ncbi.nlm.nih.gov/pubmed/16456878

18. van der Linden PD, Sturkenboom MCJM, Herings RMC, Leufkens HGM, Stricker BHC. Fluoroquinolones and risk of Achilles tendon disorders: case-control study. BMJ [Internet]. 2002 Jun 1 [cited 2015 Nov 19];324(7349):1306-7. Available from: http://www.pubmedcentral.nih.gov/articlerender.fcgi?artid $=113766 \&$ tool $=$ pmcentrez\&rendertype $=$ abstract

19. Wilson AM, Goodship AE. Exercise-induced hyperthermia as a possible mechanism for tendon degeneration. J Biomech [Internet]. 1994 Jul [cited 2015 Oct 26];27(7):899-905. Available from: http://www. ncbi.nlm.nih.gov/pubmed/8063840

20. Birch HL, Wilson AM, Goodship AE. The effect of exercise-induced localised hyperthermia on tendon cell survival. J Exp Biol [Internet]. 1997 Jun [cited 2015 Nov 19];200(Pt 11):1703-8. Available from: http://www.ncbi.nlm.nih.gov/pubmed/9202452

21. Hess GW. Achilles tendon rupture: a review of etiology, population, anatomy, risk factors, and injury prevention. Foot Ankle Spec [Internet]. 2010 Feb [cited 2015 Nov 19];3(1):29-32. Available from: http://www.ncbi.nlm.nih.gov/pubmed/20400437

22. Cary D V. How to diagnose and manage an acute Achilles tendon rupture. JAAPA [Internet]. 2009 Aug [cited 2015 Nov 19];22(8):39-43. Available from: http://www.ncbi.nlm.nih.gov/pubmed/19725413

23. Chiodo CP, Glazebrook M, Bluman EM, Cohen BE, Femino JE, Giza E, et al. Diagnosis and treatment of acute Achilles tendon rupture. J Am Acad Orthop Surg [Internet]. 2010 Aug [cited 2015 Nov 19];18(8):503-10. Available from: http://www.ncbi.nlm.nih.gov/pubmed/20675643

24. Raisbeck CC. Rupture of the Achilles tendon. J Bone Joint Surg Am [Internet]. 2000 Dec [cited 2015 Nov 19];82-A(12):1804-5. Available from: http://www.ncbi.nlm.nih.gov/pubmed/11130654

25. Martinelli B. Rupture of the Achilles tendon. J Bone Joint Surg Am [Internet]. 2000 Dec [cited 2015 Nov 19];82-A(12):1804. Available from: http://www.ncbi.nlm.nih.gov/pubmed/11130653

26. Möller M, Movin T, Granhed H, Lind K, Faxén E, Karlsson J. Acute rupture of tendon Achillis. A prospective randomised study of comparison between surgical and non-surgical treatment. J Bone Joint Surg Br [Internet]. 2001 Aug [cited 2015 Nov 19];83(6):843-8. Available from: http://www.ncbi.nlm. nih.gov/pubmed/11521926

27. Khan RJ, Carey Smith RL. Surgical interventions for treating acute Achilles tendon ruptures. Cochrane database Syst Rev [Internet]. 2010 Jan [cited 2016 Feb 11];(9):CD003674. Available from: http://www. ncbi.nlm.nih.gov/pubmed/20824836

28. Khan RJK, Fick D, Keogh A, Crawford J, Brammar T, Parker M. Treatment of acute achilles tendon ruptures. A meta-analysis of randomized, controlled trials. J Bone Joint Surg Am [Internet]. 2005 Oct [cited 2016 Jan 31];87(10):2202-10. Available from: http://www.ncbi.nlm.nih.gov/pubmed/16203884

29. Bhandari M, Guyatt GH, Siddiqui F, Morrow F, Busse J, Leighton RK, et al. Treatment of acute Achilles tendon ruptures: a systematic overview and metaanalysis. Clin Orthop Relat Res [Internet]. 2002 Jul [cited 2016 Jan 31];(400):190-200. Available from: http://www.ncbi.nlm.nih.gov/pubmed/12072762

30. Molloy A, Wood E V. Complications of the treatment of Achilles tendon ruptures. Foot Ankle Clin [Internet]. 2009 Dec [cited 2015 Nov 19];14(4):745-59. Available from: http://www.ncbi.nlm.nih.gov/ pubmed/19857846 
31. Wallace RGH, Heyes GJ, Michael ALR. The non-operative functional management of patients with a rupture of the tendo Achillis leads to low rates of re-rupture. J Bone Joint Surg Br [Internet]. 2011 Oct [cited 2016 Feb 29];93(10):1362-6. Available from: http://www.ncbi.nlm.nih.gov/pubmed/21969435

32. Jiang N, Wang B, Chen A, Dong F, Yu B. Operative versus nonoperative treatment for acute Achilles tendon rupture: a meta-analysis based on current evidence. Int Orthop [Internet]. $2012 \mathrm{Apr}$ [cited 2015 Nov 13];36(4):765-73. Available from: http://www.pubmedcentral.nih.gov/articlerender.fcgi?artid $=3311794 \&$ tool $=$ pmcentrez\&rendertype $=$ abstract

33. Soroceanu A, Sidhwa F, Aarabi S, Kaufman A, Glazebrook M. Surgical versus nonsurgical treatment of acute Achilles tendon rupture: a meta-analysis of randomized trials. J Bone Joint Surg Am [Internet]. 2012 Dec 5 [cited 2015 Aug 30];94(23):2136-43. Available from: http://www.pubmedcentral.nih.gov/ articlerender.fcgi? artid $=3509775 \&$ tool $=$ pmcentrez\&rendertype $=$ abstract

34. Maffulli N, Longo UG, Ronga M, Khanna A, Denaro V. Favorable outcome of percutaneous repair of achilles tendon ruptures in the elderly. Clin Orthop Relat Res [Internet]. 2010 Apr [cited 2016 May 7];468(4):1039-46. Available from: http://www.pubmedcentral.nih.gov/articlerender.fcgi?artid $=2835610 \&$ tool $=$ pmcentrez\&rendertype $=$ abstract

35. Maffulli N, Longo UG, Maffulli GD, Khanna A, Denaro V. Achilles tendon ruptures in diabetic patients. Arch Orthop Trauma Surg [Internet]. 2011 Jan [cited 2016 May 7];131(1):33-8. Available from: http:// www.ncbi.nlm.nih.gov/pubmed/20369360

36. McMahon SE, Smith TO, Hing CB. A meta-analysis of randomised controlled trials comparing conventional to minimally invasive approaches for repair of an Achilles tendon rupture. Foot Ankle Surg [Internet]. 2011 Dec [cited 2016 May 7];17(4):211-7. Available from: http://www.ncbi.nlm.nih.gov/ pubmed/22017889

37. Gigante A, Moschini A, Verdenelli A, Del Torto M, Ulisse S, de Palma L. Open versus percutaneous repair in the treatment of acute Achilles tendon rupture: a randomized prospective study. Knee Surg Sports Traumatol Arthrosc [Internet]. 2008 Feb [cited 2016 May 7];16(2):204-9. Available from: http:// www.ncbi.nlm.nih.gov/pubmed/18066530

38. Hüfner T, Gaulke R, Imrecke J, Krettek C, Stübig T. [Conservative functional treatment of Achilles tendon ruptures]. Unfallchirurg [Internet]. 2010 Sep [cited 2016 May 7];113(9):699-702, 704. Available from: http://www.ncbi.nlm.nih.gov/pubmed/20725820

\section{Anexos}

Tabla 1. Resultados de la estrategia de búsqueda.

\begin{tabular}{|c|c|c|c|c|}
\hline Términos de búsqueda & Medline & CINAHL & Pubmed & $\begin{array}{c}\text { Cochrane } \\
\text { Library }\end{array}$ \\
\hline $\begin{array}{c}\text { Acute achilles tendon } \\
\text { rupture }\end{array}$ & 463 & 214 & 23 & 12 \\
\hline $\begin{array}{c}\text { Achilles tendon rupture } \\
\text { treatment }\end{array}$ & 1031 & 65 & 33 & 9 \\
\hline $\begin{array}{c}\text { Achilles tendon rupture } \\
\text { conservative treatment }\end{array}$ & 156 & 27 & 6 & 1 \\
\hline $\begin{array}{c}\text { Achilles tendon rupture } \\
\text { surgical treatment }\end{array}$ & 630 & 32 & 24 & 2 \\
\hline
\end{tabular}


Tabla 2. Criterios de inclusión/exclusión.

\begin{tabular}{|c|c|}
\hline Criterios de inclusión & Criterios de exclusión \\
\hline 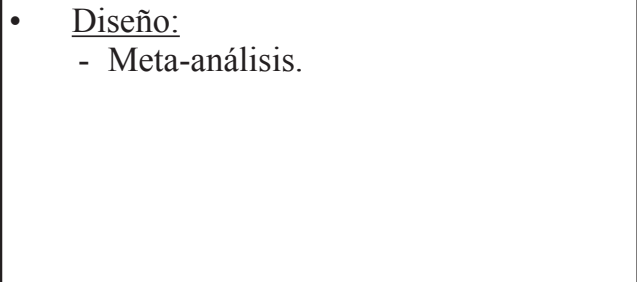 & $\begin{array}{l}\text { - Artículos que no incluyan tratamiento. } \\
\text { - Artículos no publicados en inglés. } \\
\text { - Artículos publicados hace más de } 5 \text { años. } \\
\text { - Artículos con evidencia científica limitada } \\
\text { (casos clínicos, estudios de control y } \\
\text { estudios de cohorte). } \\
\text { - Opinión del autor. }\end{array}$ \\
\hline $\begin{array}{l}\text { - Participantes: } \\
\text { - Pacientes con ruptura aguda del } \\
\text { tendón de Aquiles de cualquier causa. }\end{array}$ & \\
\hline $\begin{array}{l}\text { Actuación terapéutica: } \\
\text { - Tratamiento quirúrgico. } \\
\text { - Tratamiento conservador. }\end{array}$ & \\
\hline $\begin{array}{l}\text { - Resultados: } \\
\text { - Efectividad de los tratamientos en } \\
\text { relación a la tasa de rotura recurrente } \\
\text { y al riesgo de complicaciones. }\end{array}$ & \\
\hline
\end{tabular}

Figura 1. Revisión de la literatura.

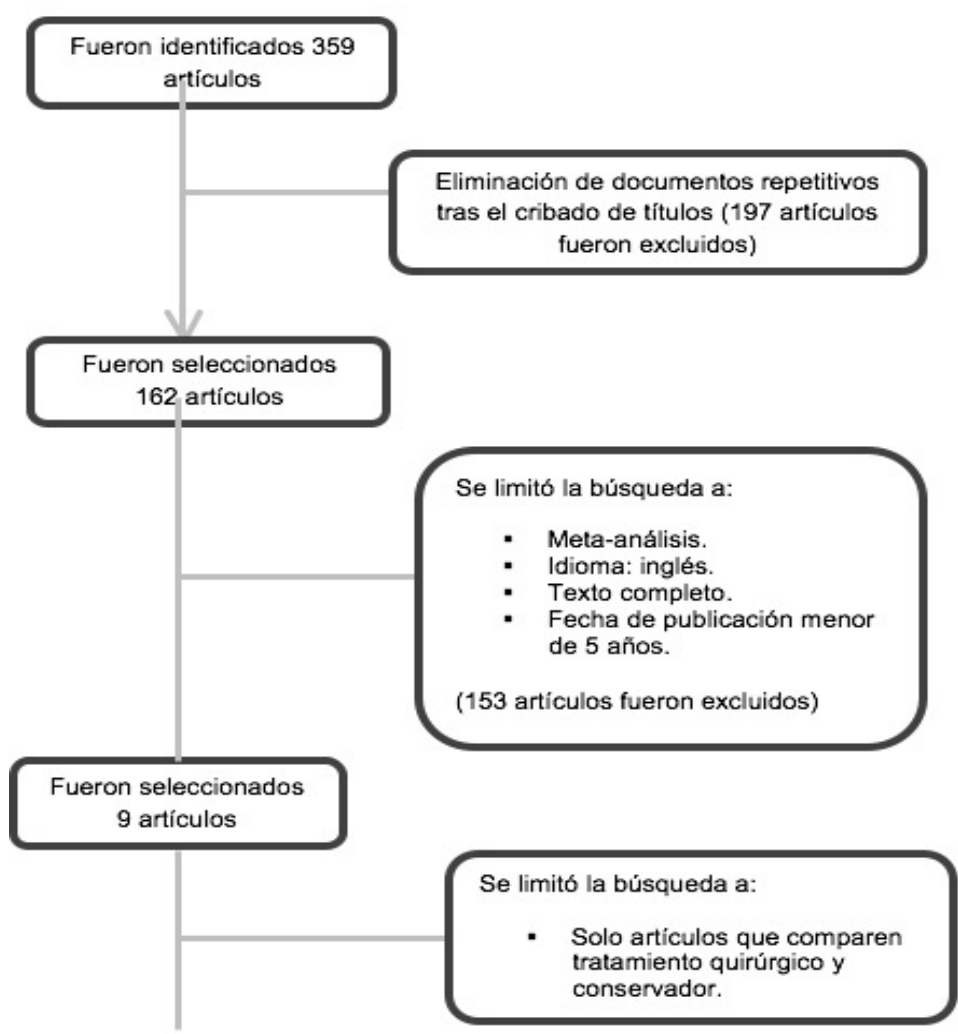

Se incluyeron 2 artículos para realizar la revisión 
Tabla 3. Resumen de los estudios incluidos.

\begin{tabular}{|c|c|c|c|c|c|}
\hline Autor, Año & $\begin{array}{l}\text { Diseño } \\
\text { estudio }\end{array}$ & $\begin{array}{l}\text { Tamaño } \\
\text { muestra }\end{array}$ & $\begin{array}{c}\text { Tipo } \\
\text { tratamiento } \\
(\mathrm{Q} / \mathrm{C})\end{array}$ & Complicaciones & $\begin{array}{c}\text { Nivel } \\
\text { evidencia }\end{array}$ \\
\hline \multirow[t]{2}{*}{$\begin{array}{l}\text { Jiang y } \\
2012(32)\end{array}$} & \multirow[t]{2}{*}{ Meta-análisis } & \multirow{2}{*}{$\begin{array}{l}\mathrm{T}=894 \\
\mathrm{TQ}=441 \\
\mathrm{TC}=453\end{array}$} & Q & $\begin{array}{l}\text { Adherencia cicatricial. } \\
\text { Infección superficial. } \\
\text { Perturbación de la } \\
\text { sensibilidad. }\end{array}$ & \multirow[t]{2}{*}{1} \\
\hline & & & $\mathrm{C}$ & $\begin{array}{l}\text { Mayor riesgo de rotura } \\
\text { recurrente. }\end{array}$ & \\
\hline \multirow[t]{2}{*}{$\begin{array}{l}\text { Soroceanu y } \\
\text { col. } 2012(33)\end{array}$} & \multirow[t]{2}{*}{ Meta-análisis } & \multirow[t]{2}{*}{$\begin{array}{l}\mathrm{T}=826 \\
\mathrm{TQ}=418 \\
\mathrm{TC}=408\end{array}$} & Q & $\begin{array}{l}\text { Infección superficial } \\
\text { y profunda, necrosis, } \\
\text { adherencia cicatricial, } \\
\text { daño nervioso, } \\
\text { disminución de la } \\
\text { movilidad, excesivo } \\
\text { alargamiento del tendón, } \\
\text { TVP. }\end{array}$ & \multirow[t]{2}{*}{1} \\
\hline & & & $\mathrm{C}$ & $\begin{array}{l}\text { Mayor tasa de re- } \\
\text { ruptura. } \\
\text { Mayor tiempo de retorno } \\
\text { al trabajo. }\end{array}$ & \\
\hline
\end{tabular}

$\mathrm{T}=$ total, $\mathrm{TQ}=$ tratamiento quirúrgico, $\mathrm{TC}=$ tratamiento conservador y $\mathrm{TVP}=$ trombosis venosa profunda. 